\title{
Coste, transporte y residuos municipales en el ámbito comarcal andaluz
}

\author{
M anuel M artín-Cobos Puebla \\ Profesor Titular de Economía Aplicada \\ Facultad de Derecho \\ Universidad de Granada ${ }^{1}$
}

RESUMEN:

La eliminación de los residuos municipales, es hoy un problema medio ambiental en todas las poblaciones de cierta entidad. Por ello, para buscar una solución en el ámbito rural, debe efectuar una opción entre las divisas técnicas existentes que den respuesta al problema planteado.

Ahora bien, todos los procedimientos para tener unos costes aceptables necesitan volúmenes de residuos municipales que sobrepasan los niveles de producción de la mayoría de los municipios rurales. De ahí la necesidad de estudiar el problema bajo la óptica de mancomunidad de municipios.

Como es fácil comprender, estas dos cuestiones -ámbito de aplicación y elección de técnica no son independientes; la ampliación del ámbito geográfico de utilización conjunta de un sistema de tratamiento permite abaratar el coste de tonelada tratada, pero, el mismo tiempo, incrementa los costes del transporte. La solución óptima, desde el punto de vista económico, será la que haga mínimos los costes globales de recogida, transporte y tratamiento.

Palabras clave: mancomunidad de municipios, minimización de costes, residuos municipales, protección medio ambiente.

${ }^{1}$ Ha colaborado bajo mi dirección en el presente trabajo, Dạ Rosa García Reina. 


\section{INTRODUCCIÓN}

La eclosión demográfica del siglo XX, la concentración de la población en zonas urbanas, el cambio en los hábitos de consumo de consumo -de lo se deriva el uso de envases sin retorno fabricados con materiales no biodegradables- y la utilización de bienes materiales de rápido envejecimiento, han traído consigo uno de los problemas más engorrosos como es el de la eliminación de las basuras.

Por ello, la actual sociedad de consumo intenta no ver sus ciudades zonas urbanas colmadas de basuras. Con el crecimiento industrial, se empezó a utilizar todo tipo de objeto y medios por un gran número de personas, por lo que los residuos urbanos crecieron de forma exponencial y pasaron a ser mezcla de desperdicios de alimentos, vidrios, plásticos, papeles, etc. con lo que se dio una dimensión superior del problema respecto de épocas anteriores.

Son múltiples las definiciones de residuo que podemos encontrar: Así pues, según la OCDE residuos son aquellas materias que generadas en las actividades de producción y consumo, no alcanzan en el contexto en el que se producen ningún valor económico, bien porque no existe una tecnología adecuada para su aprovechamiento o bien porque no existe un mercado para los productos recuperados.

Sin embargo, según la ley 10/ 1998 residuo es cualquier sustancia u objeto perteneciente a alguna de las categorías que figuran en el anejo de esta ley de la cual su poseedor se desprenda o del que tenga intención u obligación de desprenderse. En todo caso se tendrán en consideración los que figuren en el Catálogo Europeo de Residuos (CER), aprobado por las Instituciones Comunitarias. En esta definición se introduce el concepto de intencionalidad que no recogía normativas anteriores (Ley 42/ 1975 de desechos y residuos sólidos urbanos y ley 20/ 1986 básica de residuos tóxicos y peligrosos) esto implica que se considera residuo no sólo a aquel que se abandona sino también a aquel del que se tenga intención de desprenderse aunque no se haya llevado a tal efecto el abandono del mismo.

La ley 10/ 1998 de 21 de abril, de residuos deroga dos normas básicas que venían regulando hasta el año 1998 el ámbito de los residuos en nuestro país y son:

- Ley 42/ 1975 de desechos y residuos sólidos urbanos 
- Ley 20/ 1986 básica de residuos tóxicos y peligrosos

Esta ley supone la transposición de la directiva 91/ 156/ CEE, de 18 de marzo y además introduce importantes modificaciones en la ley de Bases de Régimen Local y establece que cualquier operación de gestión de residuos urbanos será competencia municipal modificando así el artículo 26 de la ley de Bases de Régimen Local según el cual solamente los municipios de más de 5000 habitantes tenían que prestar servicios obligatorios de recogida y tratamiento de residuos urbanos y los que contasen con una población inferior únicamente tendría que prestar el servicio de recogida.

Además la ley sustituye el término de residuos sólidos urbanos por el de residuos urbanos o municipales ya que algunos de estos no presentan estado sólidos. Así pues considera residuos urbanos a los siguientes:

- Los generados en domicilios particulares, oficinas, comercios y servicios.

- Los procedentes de la limpieza viaria, zonas verdes, áreas recreativas y playas.

- Los animales domésticos muertos, voluminosos y vehículos abandonados.

- Los residuos de construcción procedentes de pequeñas obras.

\section{FINALIDAD Y ARTICULACIÓN DEL ESTUDIO:}

En el presente trabajo pretendemos comparar los costes de recogida de residuos mediante dos sistemas: la carga lateral y la trasera. Partiendo del siguiente supuesto: un estado óptimo donde se hace una buena segregación de los residuos urbanos que implica que en el contenedor gris solamente tendríamos materia orgánica.

Según el Plan Nacional de Residuos U rbanos (BOE de 2 de febrero de 2000) la generación de residuos urbanos se cifra en $1.2 \mathrm{Kg} /$ habitante/ día y la composición media de los residuos urbanos para el periodo 1991-1996 es la siguiente: 
DOCUMENTOS

\begin{tabular}{|l|c|}
\hline \multicolumn{2}{|c|}{ TABLA 2.1 } \\
\hline COMPONENTE & $\begin{array}{c}\text { COMPOSICIÓ N MEDIA (1991-6) } \\
(\%)\end{array}$ \\
\hline Materia orgánica & 44.06 \\
\hline Papel-cartón & 21.18 \\
\hline Plástico & 10.59 \\
\hline Vidrio & 6.93 \\
\hline Metales & 4.11 \\
\hline Maderas & 0.96 \\
\hline Otros & 12.17 \\
\hline FUENTE: Plan Nacional de Residuos U rbanos (2000). Elaboración propia. \\
\hline
\end{tabular}

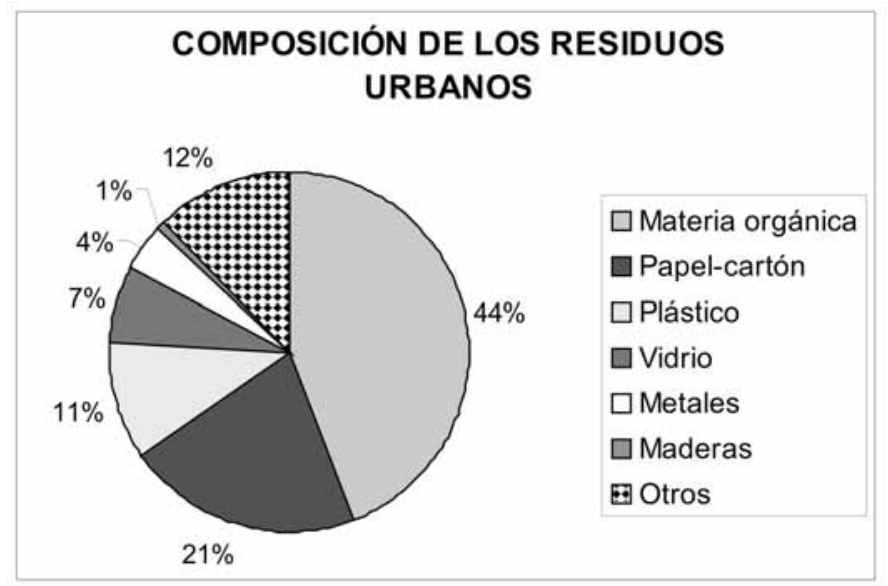

FUENTE: Plan Nacional de Residuos Urbanos (2000). Elaboración propia.

Así pues, partiendo de estos datos podemos calcular la generación de materia orgánica que es $0.53 \mathrm{Kg} /$ habitante/ día. 


\section{IDENTIFICACIÓN DE LA COMARCA OBJETO DE ESTUDIO.}

En el presente estudio trabajaremos con la comarca de la provincia de Jaén compuesta por los siguientes municipios:

- bAEZA

- begíjar

- cANENA

- IBROS

- LUPIÓN

- RUS

- sabiote

- TORRE PEROGIL

- ÚBEDA

Para comenzar es importante conocer la demografía de la comarca al objeto de prever la evolución de la población durante el periodo de tiempo que será objeto de estudio.

TABLA 3.1

EVOLUCIÓN DE LA POBLACIÓN EN LA COMARCA DE ESTUDIO DURANTE EL PERIODO 1950-2004

\begin{tabular}{|l|r|r|r|r|r|r|r|r|r|}
\hline \multicolumn{10}{|c|}{ AÑO } \\
\hline & 1950 & 1960 & 1970 & 1979 & 1989 & 1995 & 2000 & 2002 & 2004 \\
\hline BAEZA & 16895 & 15461 & 14834 & 15581 & 15086 & 15900 & 15738 & 15223 & 15177 \\
\hline BEGÍJAR & 4385 & 4371 & 3319 & 3263 & 3160 & 3174 & 3136 & 3117 & 3109 \\
\hline CANENA & 2430 & 2457 & 2100 & 2079 & 2138 & 2117 & 2122 & 2132 & 2103 \\
\hline IBROS & 4884 & 4544 & 3909 & 3339 & 3294 & 3225 & 3015 & 3060 & 3021 \\
\hline LUPIÓN & 1241 & 1651 & 1600 & 1293 & 1247 & 1111 & 1050 & 1054 & 1026 \\
\hline RUS & 4508 & 4526 & 3830 & 3863 & 3737 & 3826 & 3747 & 3769 & 3743 \\
\hline SABIOTE & 7360 & 6887 & 5053 & 5142 & 4519 & 4333 & 4192 & 4194 & 4141 \\
\hline TORRE PEROGIL & 10146 & 9606 & 8528 & 9664 & 7801 & 7964 & 7424 & 7227 & 6948 \\
\hline ÚBEDA & 30249 & 28956 & 30186 & 32669 & 31344 & 31819 & 32456 & 32971 & 33511 \\
\hline TOTAL & $\mathbf{8 2 0 9 8}$ & $\mathbf{7 8 4 5 9}$ & $\mathbf{7 3 3 5 9}$ & $\mathbf{7 6 8 9 3}$ & $\mathbf{7 2 3 2 6}$ & $\mathbf{7 3 4 6 9}$ & $\mathbf{7 2 8 8 0}$ & $\mathbf{7 2 7 4 7}$ & $\mathbf{7 2 7 7 9}$ \\
\hline
\end{tabular}

FUENTES: Padrón de municipal. INE. Nomeclator. INE. Sistema de información multiterritorial. IEA. Elaboración propia. 
Considerando que se mantienen las tendencias actuales en relación a la demografía de los núcleos de estudio haremos una estimación de población para los próximos 10 años periodo de vida útil de la instalación de recepción de los residuos. En principio se pensó determinar la ubicación de un vertedero o una estación de transferencia pero dado que en nuestro supuesto solo realizamos una recogida de materia orgánica lo que calcularemos en los siguientes apartados de este estudio será la ubicación de una planta de compostaje.

Para llevar a cabo la estimación anteriormente mencionada calcularemos el crecimiento medio anual de los municipios de la comarca a través de la siguiente formula:

CRECIMIENTO MEDIO ANUAL $=100 \times$ ( ( población final - población inicial)/ población media del periodo considerado)/ $n$ o de años del periodo

TABLA 3.2

\section{TABLA DE CÁLCULO DEL CRECIMIENTO MEDIO ANUAL DE LA POBLACIÓN EN LA COMARCA DE ESTUDIO DURANTE EL PERIODO 1989-2004.}

\begin{tabular}{|c|c|c|c|c|c|c|c|}
\hline & \multicolumn{5}{|c|}{ AÑO } & \multirow[b]{2}{*}{$\begin{array}{r}\text { POBLACIÓN } \\
(1989-2004)\end{array}$} & \multirow[b]{2}{*}{$\begin{array}{r}\text { Crecimiento } \\
\text { medio anual } \\
(\%)\end{array}$} \\
\hline & 1989 & 1995 & 2000 & 2002 & 2004 & & \\
\hline BAEZA & 15086 & 15900 & 15738 & 15223 & 15177 & 15413 & 0.039 \\
\hline BEGÍJAR & 3160 & 3174 & 3136 & 3117 & 3109 & 3139 & -0.108 \\
\hline CANENA & 2138 & 2117 & 2122 & 2132 & 2103 & 2122 & -0.12 \\
\hline IBROS & 3294 & 3225 & 3015 & 3060 & 3021 & 3123 & -0.583 \\
\hline LUPIÓN & 1247 & 1111 & 1050 & 1054 & 1026 & 1098 & -1.342 \\
\hline RUS & 3737 & 3826 & 3747 & 3769 & 3743 & 3764 & 0.011 \\
\hline SABIOTE & 4519 & 4333 & 4192 & 4194 & 4141 & 4276 & -0.589 \\
\hline TORRE PEROGIL & 7801 & 7964 & 7424 & 7227 & 6948 & 7473 & -0.761 \\
\hline ÚBEDA & 31344 & 31819 & 32456 & 32971 & 33511 & 32420 & 0.446 \\
\hline TOTAL & 72326 & 73469 & 72880 & 72747 & 72779 & 72840 & 0.041 \\
\hline
\end{tabular}

FUENTES: Padrón de municipal. INE. Nomeclator. INE. Sistema de información multiterritorial. IEA. Elaboración propia. 


\section{LOCALIZACIÓN DEL CENTRO DE TRATAMIENTO}

Para ubicar una planta de compostaje que ha sido el centro de tratamiento elegido en nuestro estudio debemos tener en cuenta una serie de factores según HONTORIA de tipo ambiental y económico ${ }^{1}$. Así pues la ubicación elegida deberá cumplir los siguientes criterios de ubicación:

- Ser un zona estratégica respecto a la ubicación de las poblaciones productoras de residuos y tener asegurada la venta de compost en un radio inferior a $100 \mathrm{~km}$.

- Deberá de situarse fuera de los núcleos de población y espacios naturales protegidos así como de sus zonas de influencia.

- Se deberá realizar un estudio litológico e hidrológico del terreno con el fin de determinar la ubicación de esta instalación en terrenos impermeables o de baja permeabilidad y con ausencia de acuíferos de entidad.

- Si se quiere rentabilizar la planta de compostaje debe recoger la materia orgánica de una comarca con población superior a los 100000 habitantes.

Dada la importancia de los costes del transporte en el coste de total del servicio es preciso hallar una ubicación que además de cumplir con los criterios anteriores minimice los costes en el transporte. En este estudio únicamente se tendrán en cuenta que la ubicación de la planta de compostaje minimice los costes del transporte es decir que la suma de las distancias de la planta de compostaje a los distintos municipios a los que se presta el servicio sea mínima. Para establecer la ubicación optima existen distintos procedimientos como:

- La matriz de distancias mínimas

- El vector de producción y matriz de distancias físicas

- Modelo de gravedad

\section{Procedimiento 1: matriz de distancias mínimas}

Este método consiste en obtener las distancias mínimas de cada municipio al resto de municipios que integran la comarca de estudio. Para ello se han calculado las distancias mínimas mediante un mapa oficial de carreteras de Andalucía de la serie provincial de Granada a escala 1:200000 editado en el año 2003 por la Consejería de Obras Públicas y Transportes. 
TABLA 4.1

Tabla de distancias mínimas (km) de los municipios de la comarca de estudio

\begin{tabular}{|l|r|r|r|r|r|r|r|r|r|}
\hline & BAEZA & BEGIJAR & CANENA & IBROS & LUPIÓN & RUS & SABIOTE & TORRE & ÚBEDA \\
& & & & & & & & & \\
\hline BAEZA & 0 & 6 & 9.5 & 3 & 6.5 & 6.5 & 15.5 & 15 & 6.5 \\
\hline BEGIJAR & 6 & 0 & 11.5 & 5 & 2 & 12.5 & 24.5 & 24 & 17.5 \\
\hline CANENA & 9.5 & 11.5 & 0 & 6.5 & 12 & 2 & 18 & 17.5 & 11 \\
\hline IBROS & 3 & 5 & 6.5 & 0 & 5.5 & 8.5 & 24.5 & 18.5 & 9.5 \\
\hline LUPIÓN & 6.5 & 2 & 12 & 5.5 & 0 & 12 & 27 & 32 & 18 \\
\hline RUS & 6.5 & 12.5 & 2 & 8.5 & 12 & 0 & 16 & 15.5 & 11 \\
\hline SABIOTE & 15.5 & 24.5 & 18 & 24.5 & 27 & 16 & 0 & 3.5 & 8 \\
\hline TORRE PEROGIL & 15 & 24 & 17.5 & 18.5 & 32 & 15.5 & 3.5 & 0 & 6.5 \\
\hline ÚBEDA & 6.5 & 17.5 & 11 & 9.5 & 18 & 11 & 8 & 6.5 & 0 \\
\hline TOTAL & 68.5 & 85.5 & 88 & 81 & 115 & 84 & 137 & 147.5 & 88 \\
\hline
\end{tabular}

FUENTE: Mapa oficial de carreteras de Andalucía. Serie provincial de Granada. Consejería de Obras Públicas y Transportes. (2003). Elaboración propia.

Según los resultados presentados en la tabla la ubicación optima para la planta de compostaje estaría en el término municipal de Baeza.

\section{Procedimiento 2: vector de producción y matriz de distancias físicas}

En este procedimiento introducimos una nueva variable que es el vector de producción de residuos municipal. Multiplicando el vector de producción por la matriz de distancias físicas podremos determinar el municipio donde se ubicará la planta de compostaje. Para desarrollar los cálculos de este método utilizaremos la siguiente expresión:

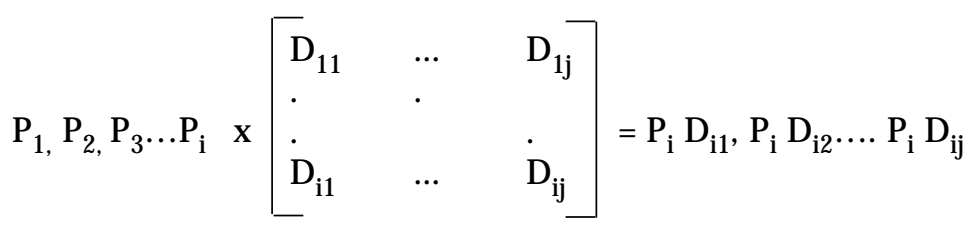

$\mathrm{P}_{\mathrm{i}}=$ producción de residuos de cada municipio.

$\mathrm{D}_{\mathrm{ij}}=$ Distancia del municipio considerado a cada uno de los restantes. 
Así pues, partiendo de la tabla 4.1 y multiplicando por el vector de producción de residuos municipal obtenemos la siguiente tabla:

\section{TABLA 4.2}

Tabla resultante de la aplicación del procedimiento 2 para la obtención la ubicación que minimice los coste del transporte de residuos en la comarca de estudio.

\begin{tabular}{|c|c|}
\hline & $\Sigma P_{i}{ }^{*} D_{i j}$ \\
\hline ÚBEDA & $\mathbf{1 8 1 6 9 5 , 3 9 5}$ \\
\hline BAEZA & 246408,13 \\
\hline IBROS & 350092,03 \\
\hline RUS & 379433,63 \\
\hline TORRE PEROGIL & 380606,52 \\
\hline CANENA & 415582,81 \\
\hline SABIOTE & 425737,61 \\
\hline BEGÍJAR & 547935,20 \\
\hline LUPIÓN & 598357,81 \\
\hline
\end{tabular}

FUENTE: Mapa oficial de carreteras de Andalucía. Serie provincial de Granada. Consejería de Obras Públicas y Transportes. (2003). Elaboración propia.

NOTA: Los valores $\Sigma P_{i} * D_{i j}$ están expresados en $\mathrm{Kg} \times \mathrm{Km}$ al día.

Según los resultados de la tabla 4.2 el municipio que minimiza los costes de transporte es Úbeda y por tanto según este procedimiento la planta de compostaje debería de ubicarse en esta localidad.

\section{Procedimiento 3: Modelo de gravedad}

En este procedimiento calculamos el centro de gravedad ponderado que es aquel punto en el que cuando aplicamos de una fuerza vertical podemos equilibrar todas las fuerza de gravedad que actúan sobre un cuerpo. 


\section{TABLA 4.3}

Tabla resultante de la aplicación del modelo de gravedad para la obtención la ubicación que minimice los coste del transporte de residuos en la comarca de estudio.

\begin{tabular}{|l|r|r|r|r|r|}
\hline & $X_{i}$ (metros) & $Y_{i}$ (metros) & $\begin{array}{r}P_{i} \text { (población } \\
\text { 2004) }\end{array}$ & $P_{i} X_{i}$ & $P_{i} Y_{i}$ \\
\hline BAEZA & 384.9 & 4216.55 & 15177 & 5841627.3 & 63994579.35 \\
\hline BEGÍJAR & 391.2 & 4214.75 & 3109 & 126240.8 & 13103657.75 \\
\hline CANENA & 386.4 & 4222.33 & 2103 & 812599.2 & 8879559.99 \\
\hline IBROS & 389 & 4219.54 & 3021 & 1175169 & 12747230.34 \\
\hline LUPIÓN & 393.5 & 4216.55 & 1026 & 403731 & 4326180.3 \\
\hline RUS & 383.9 & 4222.33 & 3743 & 146937.7 & 15804181.19 \\
\hline SABIOTE & 367.5 & 4225.33 & 4141 & 1521817.5 & 17497091.53 \\
\hline TORRE PEROGIL & 365 & 4221.34 & 6948 & 2536020 & 29329870.32 \\
\hline ÚBEDA & 374 & 4218.54 & 33511 & 12533114 & 141367559.9 \\
\hline TOTAL & & & 72779 & 27477256.5 & $\mathbf{3 0 7 0 4 9 9 1 0 . 7}$ \\
\hline
\end{tabular}

FUENTE: Mapa oficial de carreteras de Andalucía. Serie provincial de Granada. Consejería de O bras Públicas y Transportes. (2003). Elaboración propia.

U na vez localizadas las coordenadas geográficas de los distintos municipios en el mapa hemos pasado a metros dichas coordenadas mediante la siguiente equivalencia:

$$
1 \text { segundo }=30.83 \text { metros }
$$

A continuación hemos aplicado las siguientes expresiones para obtener el centro de gravedad.

Latitud $u$ ordenada, $X=\Sigma X_{i} P_{i} / S P_{i}$

Longitud 0 abcisa, $Y=\Sigma Y_{i} P_{i} / S P_{i}$ 
$Y_{\mathrm{i}}=$ longitudes geográficas

$X_{i}=$ latitudes geográficas

$\mathrm{P}_{\mathrm{i}}=$ ponderación de cada municipio

Así pues, las coordenadas del centro de gravedad son:

$X=3024^{\prime} 0^{\prime \prime}$

$\mathrm{Y}=38^{\circ} 00^{\prime} 44.96^{\prime \prime}$

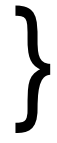

El centro de gravedad se halla situado en Úbeda.

\section{CÁLCULO DE NECESIDADES}

El cálculo de la capacidad total lo realizaremos en función de la evolución de la producción de residuos durante el periodo de vida de la planta de compostaje. En este estudio hemos tenido en cuenta solamente la evolución de la población para el cálculo de la evolución de la producción de residuos. El cálculo de dicha evolución se ha realizado a través de la siguiente expresión:

$$
\mathrm{P}_{\mathrm{T}}=\mathrm{P}_{\mathrm{A}}\left((1+\mathrm{r})^{\mathrm{n}}-1\right) / \mathrm{r}
$$

$\mathrm{P}_{\mathrm{A}}=$ producción en el año 2004.

$\mathrm{P}_{\mathrm{T}}=$ producción total de residuos orgánicos en el periodo 2004-14.

$r=$ tasa de crecimiento anual de los residuos orgánicos.

$\mathrm{n}=$ vida útil de la planta de compostaje ( se ha estimado en 10 años).

TABLA 5.1

Tabla de cálculo de producción total de residuos orgánicos en la comarca de estudio durante el periodo 2004-14. 


\begin{tabular}{|l|c|c|c|c|c|c|}
\hline & $\begin{array}{c}\text { Población } \\
\text { en 2004 }\end{array}$ & $\begin{array}{c}\text { tasa } \\
\text { crecimiento } \\
(\%)\end{array}$ & $r$ & $\mathrm{P}_{\mathrm{A}}$ & $\mathrm{P}_{\mathrm{T}}$ (2004-14) & $\begin{array}{c}\text { Producción } \\
\text { media anual } \\
\text { para el } \\
\text { período } \\
2004 / 14\end{array}$ \\
\hline BAEZA & 15177 & 0,039 & 0,0002067 & 8043,81 & 80512,9608 & 8051,296075 \\
\hline BEGÍJAR & 3109 & $-0,108$ & $-0,0005724$ & 1647,77 & 16435,3215 & 1643,532146 \\
\hline CANENA & 2103 & $-0,12$ & $-0,000636$ & 1114,59 & 11114,0545 & 1111,405448 \\
\hline IBROS & 3021 & $-0,583$ & $-0,0030899$ & 1601,13 & 15790,4946 & 1579,049461 \\
\hline LUPIÓN & 1026 & $-1,342$ & $-0,0071126$ & 543,78 & 5267,01435 & 526,7014346 \\
\hline RUS & 3743 & 0,011 & 0,0000583 & 1983,79 & 19843,1053 & 1984,310528 \\
\hline SABIOTE & 4141 & $-0,589$ & $-0,0031217$ & 2194,73 & 21641,5446 & 2164,154456 \\
\hline TORRE PEROGIL & 6948 & $-0,761$ & $-0,0040333$ & 3682,44 & 36163,1807 & 3616,318067 \\
\hline ÚBEDA & 33511 & 0,446 & 0,0023638 & 17760,83 & 179509,495 & 17950,94954 \\
\hline TOTAL & 72779 & 0,041 & 0,0002173 & 38572,87 & 386106,103 & 38610,61035 \\
\hline
\end{tabular}

FUENTE: Padrón municipal de población (2004). Elaboración propia.

\section{PLANTEAMIENTO TEÓRICO Y CÁLCULOS}

a) CÁLCULO DE LA DISTANCIA NECESARIA A RECORRER PARA TRANSPORTAR LA TOTALIDAD DE LOS RESIDUOS A LA PLANTA DE COMPOSTAJE.

$\mathrm{K}_{\mathrm{i}}=$ distancia en $\mathrm{km}$ desde el municipio a la planta de compostaje

$\mathrm{N}_{\mathrm{i}}=$ número de contenedores necesarios

$\mathrm{V}_{\mathrm{i}}=$ volumen que ocupan los contenedores sin compactar

$M=$ volumen del contenedor

$Z_{i}=$ peso en $\mathrm{kg}$. de los residuos

$X=$ densidad de los residuos $\left(\mathrm{Kg} / \mathrm{m}^{3}\right)$

$\mathrm{D}_{\mathrm{i}}=$ distancia a recorrer

$$
\begin{gathered}
D_{i}=Z_{i} N_{i} K_{i} \\
N_{i}=V_{i} / M \\
V_{i}=Z_{i} / X
\end{gathered}
$$

Sustituyendo obtenemos:

$$
\begin{gathered}
N_{i}=Z_{i} /\left(X_{i} M\right) \\
D_{i}=\left(2 K_{i} Z_{i}\right) /\left(X_{i} M\right)
\end{gathered}
$$


Los residuos que se recogen son como ya he comentado materia orgáni$\mathrm{ca}$, es decir, fundamentalmente restos de comida cuya densidad varia en función de la humedad de los residuos y GARCíA RAMOS (1996) cifra en 131 $\mathrm{Kg} / \mathrm{m}^{3}$ para un grado de humedad del $50 \%{ }^{2}$.

Para simplificar la siguiente expresión $D_{i}=\left(2 K_{i} Z_{i}\right) /\left(X_{i} M\right)$ denotamos con $\mathrm{F} \mathrm{a}\left(2 \mathrm{~K}_{\mathrm{i}} \mathrm{Z}_{\mathrm{i}}\right)$ por tanto la expresión de la distancia a recorrer nos queda de la siguiente forma:

$$
D_{i}=F /\left(X_{i} M\right)
$$

Si $\mathrm{X}$ y $\mathrm{F}$, es decir, la densidad de los residuos y el producto de la distancia de a la planta de compostaje por el número de contendores del municipio considerado son constantes, por tanto lo que nos varia en la siguiente expresión es el volumen del contenedor (M). Por tanto, podemos considerar $D_{i}$ (distancia a recorrer) como una función de M (volumen del contenedor) Cuya representación gráfica es la siguiente:

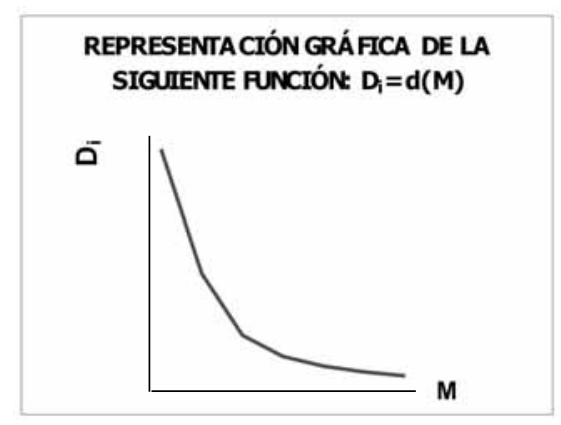

La función $D_{i}=d(M)$, es decreciente ya que conforme aumenta el volumen del contenedor el número de trayectos y por tanto la distancia a recorrer para transportar la totalidad de los residuos a la planta de compostaje $D_{i}$ es menor.

b) CÁLCULO DE LA DISTANCIA MÁXIMA A RECORRER POR UN VEHÍCULO DURANTE LA JORNADA LABORAL

$\mathrm{V}_{\mathrm{m}}=$ velocidad media del vehículo en $\mathrm{km} / \mathrm{h}$

$\mathrm{T}=$ tiempo perdido en carga y descarga y otras eventualidades

$\mathrm{g}=$ perdida total de kilómetros 
$2 \mathrm{~N}_{\mathrm{i}}=$ número de operaciones de carga y descarga boral

$\mathrm{D}_{\max }=$ distancia máxima a recorrer por el vehículo durante la jornada la-

$$
\begin{gathered}
g=2 N_{i} T^{\top} \\
N_{i}=Z_{i} l\left(X_{i} M\right)
\end{gathered}
$$

Sustituyendo obtenemos:

$$
\mathrm{g}=\left(2 \mathrm{~T} \mathrm{~V}_{\mathrm{m}} \mathrm{Z}_{\mathrm{i}}\right) /(\mathrm{X} \mathrm{M})
$$

A partir de la expresión anterior y la siguiente:

$$
\begin{gathered}
\mathrm{D}_{\text {max }}=5.5 \mathrm{~V}_{\mathrm{m}}-\mathrm{g} \\
\text { Tenemos que: } \\
\mathrm{D}_{\max }=5.5 \mathrm{~V}_{\mathrm{m}}-\left(2 \mathrm{~T} \mathrm{~V}_{\mathrm{m}} \mathrm{Z}_{\mathrm{i}}\right) /(\mathrm{X} \mathrm{M})
\end{gathered}
$$

La jornada laboral del personal de recogida de residuos es 35 horas semanales ${ }^{3}$ lo que supone 6 jornadas de unas 6 horas. Para el cálculo de la distancia máxima que puede recorrer un vehículo hemos tenido en cuenta una jornada de 5.5 horas ya que ha esta se le ha restado con anterioridad el tiempo correspondiente al descanso del personal. En cuanto a la velocidad media de los camiones tenemos que indicar que normal mente los fabricante limitan la velocidad del camión a $90 \mathrm{~km} / \mathrm{h}^{4}$ y que en el caso de no ser así algunas empresa como CESPA-INGENIERÍA URBANA limitan la velocidad de su camión estableciendo como valor máximo el anteriormente indicado. Así pues la velocidad media en carretera es de $75 \mathrm{~km} / \mathrm{h}$ y en núcleo urbano de $15 \mathrm{~km} / \mathrm{h}$.

Para simplificar la siguiente expresión $D_{\max }=5.5 \mathrm{~V}_{m}-\left(2 \mathrm{~T} \mathrm{~V}_{m} \mathrm{Z}_{\mathrm{i}}\right) /(\mathrm{XM})$ denotamos como $B$ a $\left(2 T V_{m} Z_{i}\right)$ obtenemos que $D_{\max }=5.5 V_{m}-B /(X M)$.

Si $V_{m}$ y $B$, es decir, la velocidad media del VEH ÍCULO y el producto de la misma por la perdida de tiempo en cargar y descarga y otras eventualidades y por la distancia de a la planta de compostaje del municipio considerado son constantes, por tanto lo que nos varia en la siguiente expresión es el volumen del contenedor ( $M$ ). Así pues, podemos considerar $D_{\max }$ (distancia máxima a recorrer por el vehículo durante la jornada laboral) como una función de M (volumen del contenedor) cuya representación gráfica es la siguiente: 


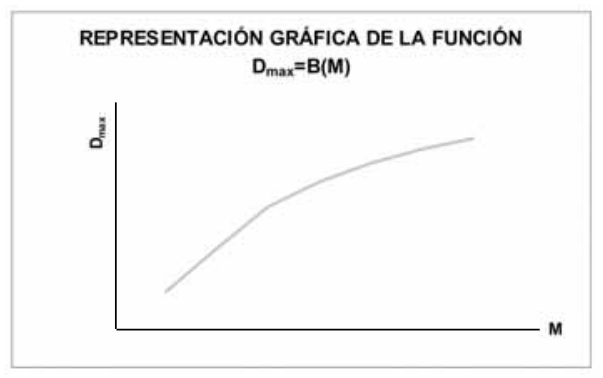

La representación de la función $D_{\max }=B(M)$, es creciente ya que conforme aumenta el volumen del contenedor $(M)$ el número de cargas y descargas así como el tiempo perdido será mayor y por tanto será mayor la distancia a recorrer durante la jornada laboral $\left(D_{\max }\right)$.

Si a continuación representamos tal y como muestra la siguiente gráfica las funciones $D_{\max }=B(M)$ y $D_{i}=d(M)$ en un mismo gráfico podemos hallar la situación de equilibrio en el punto de intersección de ambas.

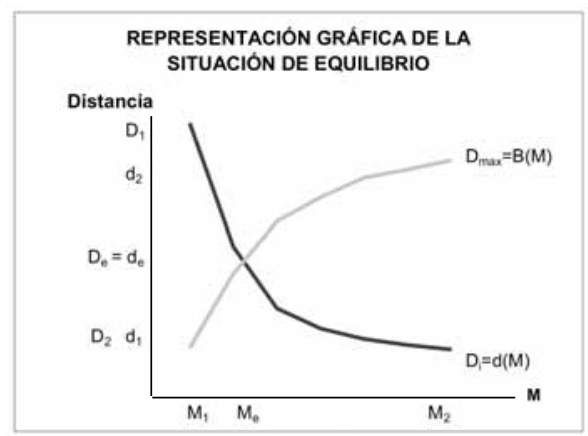

Según el gráfico de la representación de la situación de equilibrio podemos encontrar 3 casos:

Caso 1: Para un volumen de contendor pequeño $\left(\mathrm{M}_{1}\right)$ la distancia a recor rer $\left(D_{1}\right)$ es superior a la distancia máxima que puede recorrer un vehículo durante la jornada laboral $\left(d_{1}\right)$, por tanto necesitaríamos varios vehículos, de tal modo que el número de vehículos (C) vendría determinado por la siguiente expresión:

$$
C=D_{1} / d_{1}
$$


$\mathrm{C}=$ número de vehículos

$\mathrm{D}_{1}=$ la distancia a recorrer ral

$\mathrm{d}_{1}=$ distancia máxima recorrida por un vehículo durante la jornada labo-

Caso 2: Para un volumen de contendor grande $\left(\mathrm{M}_{2}\right)$ la distancia a reco$\operatorname{rrer}\left(D_{2}\right)$ es inferior a la distancia máxima que puede recorrer un vehículo durante la jornada laboral $\left(d_{2}\right)$, por tanto el vehículo permanecería ocioso durante el tiempo que se tarda en recorrer $d_{2}-D_{2}$.

Caso 3: Para un volumen de contendor $\left(\mathrm{M}_{\mathrm{e}}\right)$ se alcanza la situación de equilibrio ya que la distancia a recorrer para transportar los residuos a la planta de compostaje $\left(D_{\mathrm{e}}\right)$ es igual a la distancia máxima que puede recorrer un vehículo durante la jornada laboral $\left(\mathrm{d}_{\mathrm{e}}\right)$.

\section{RECOGIDA MEDIANTE CAMIONES DE CARGA LATERAL}

En este apartado hemos trabajado con contenedores de 2400 litros y los datos correspondientes al siguiente camión ${ }^{5}$ :

$$
\begin{aligned}
& \text { MODELO FMEO } 25 \\
& \text { Volumen de caja }=25 \mathrm{~m}^{3} \\
& \text { Compactación } 6: 1 \\
& \text { Carga útil }=13-14 \mathrm{Tm} \\
& \text { Tiempo de carga }=40 \text { segundos } \\
& \text { Precio }=180000 €
\end{aligned}
$$

Al tiempo le hemos agregado un minuto para tener en cuenta las maniobras que realiza el camión para recoger los contenedores y otras eventualidades.

\section{TABLA 6.1}

Tabla de resultante de la aplicación de las expresiones recogidas en los apartados

6.a. y 6.b. para cada uno del los municipio de la comarca de estudio en el caso de que la recogida de residuos se efectúe mediante camiones de carga lateral. 


\begin{tabular}{|c|c|c|c|c|c|c|c|}
\hline & $\mathrm{K}_{\mathrm{i}}$ & $z_{i}$ & $v_{i}$ & $\mathrm{~N}_{\mathrm{i}}$ & $\begin{array}{r}\mathrm{n} 0 \\
\text { contenedores }\end{array}$ & $D_{i}$ & $D_{\max }$ \\
\hline BAEZA & 6,5 & 8051,3 & 61,4603053 & 25,6084606 & 31 & 332,909987 & 177,3 \\
\hline BEGÍJAR & 17,5 & 1643,53 & 12,5460305 & 5,22751272 & 7 & 182,962945 & 231,3 \\
\hline CANENA & 11 & 1111,4 & 8,48396947 & 3,53498728 & 5 & 77,7697201 & 236,7 \\
\hline IBROS & 9,5 & 1579,05 & 12,0538168 & 5,02242366 & 7 & 95,4260496 & 231,3 \\
\hline LUPIÓN & 18 & 526,7 & 4,02061069 & 1,67525445 & 2 & 60,3091603 & 242,1 \\
\hline RUS & 11 & 1984,31 & 15,1474046 & 6,31141858 & 8 & 138,851209 & 228,6 \\
\hline SABIOTE & 8 & 2164,15 & 16,520229 & 6,88342875 & 8 & 110,13486 & 228,6 \\
\hline TORRE PEROGIL & 6,5 & 3616,32 & 27,6054962 & 11,5022901 & 14 & 149,529771 & 215,1 \\
\hline ÚBEDA & 0 & 17950,95 & 137,030153 & 57,0958969 & 71 & 0 & 88,2 \\
\hline TOTAL & 88 & 38610,61 & 294,737481 & 122,807284 & 153 & 21614,0819 & $-100,8$ \\
\hline
\end{tabular}

FUENTE: Tablas 4.1 y 5.1 del presente estudio. Elaboración propia.

Según lo observado en la tabla 6.1 la distancia a recorrer en Ú beda es 0 $\mathrm{km}$ esto se debe ha que se ha establecido este municipio como punto de localización de la planta de compostaje y no se tenido en cuenta que para recoger los 71 contenedores que poseen debe de realizarse un recorrido intraurbano que estimaremos en función de la distancia a recorrer en Baeza en 665.82 km.

\section{RECOGIDA MEDIANTE CAMIONES DE CARGA TRASERA}

En este apartado hemos trabajado con contenedores de 1100 litros y los datos correspondientes al siguiente camión ${ }^{6}$ :

\begin{tabular}{l}
\hline MODELO RSE-CROSS 21 \\
Volumen de caja $=21 \mathrm{~m}^{3}$ \\
Compactación $6: 1$ \\
Carga útil $=9-10 \mathrm{Tm}$ \\
Tiempo de carga $=20$ segundos \\
Precio $=120000 €$
\end{tabular}

Al tiempo le hemos agregado 2 minutos para tener en cuenta las maniobras que realiza el camión para recoger los contenedores, el tiempo que los operarios tardan en colocar el contenedor y otras eventualidades. 


\section{TABLA 6.2}

Tabla de resultante de la aplicación de las expresiones recogidas en los apartados 6.a. y 6.b. para cada uno del los municipio de la comarca de estudio en el caso de que la recogida de residuos se efectúe mediante camiones de carga lateral.

\begin{tabular}{|l|r|r|r|r|r|r|r|}
\hline & \multicolumn{1}{|c|}{$\mathrm{K}_{\mathrm{i}}$} & \multicolumn{1}{c}{$\mathrm{Z}_{\mathrm{i}}$} & \multicolumn{1}{c|}{$\mathrm{V}_{\mathrm{i}}$} & $\mathrm{N}_{\mathrm{i}}$ & $\begin{array}{r}\mathrm{n} 0 \\
\text { contenedores }\end{array}$ & $\mathrm{D}_{\mathrm{i}}$ & $\mathrm{D}_{\max }$ \\
\hline BAEZA & 6,5 & 8051,3 & 61,4603053 & 55,8730049 & 67 & 726,349063 & 20,7 \\
\hline BEGÍJAR & 17,5 & 1643,53 & 12,5460305 & 11,4054823 & 14 & 399,191881 & 198,9 \\
\hline CANENA & 11 & 1111,4 & 8,48396947 & 7,71269951 & 9 & 169,679389 & 215,1 \\
\hline IBROS & 9,5 & 1579,05 & 12,0538168 & 10,9580153 & 13 & 208,20229 & 202,95 \\
\hline LUPIÓN & 18 & 526,7 & 4,02061069 & 3,65510062 & 4 & 131,583622 & 231,3 \\
\hline RUS & 11 & 1984,31 & 15,1474046 & 13,7703678 & 16 & 302,948092 & 190,8 \\
\hline SABIOTE & 8 & 2164,15 & 16,520229 & 15,01839 & 19 & 240,29424 & 182,7 \\
\hline TORRE PEROGIL & 6,5 & 3616,32 & 27,6054962 & 25,0959056 & 30 & 326,246773 & 146,25 \\
\hline ÚBEDA & 0 & 17950,95 & 137,030153 & 124,572866 & 150 & & $-258,75$ \\
\hline TOTAL & 88 & 38610,61 & 294,737481 & 267,943164 & 322 & 47157,9969 & $-850,05$ \\
\hline
\end{tabular}

FUENTE: Tablas 4.1 y 5.1 del presente estudio. Elaboración propia.

Según lo observado en la tabla 6.2 la distancia a recorrer en Ú beda es 0 km esto se debe ha que se ha establecido este municipio como punto de localización de la planta de compostaje y no se tenido en cuenta que para recoger los 150 contenedores que poseen debe de realizarse un recorrido intraurbano que estimaremos en función de la distancia a recorrer en Baeza en 1452.70 km.

\section{ORGANIZACIÓN DEL SERVICIO DE RECOGIDA SEGÚN LA OPCIÓN DEL CONTENEDOR}

Basándonos en las argumentaciones teóricas expuestas en apartados anteriores a continuación procederemos a agrupar el servicio de recogida en distintos circuitos para cada tipo de recogida (mediante carga lateral y trasera) estableciendo los horarios de recogida y los vehículos necesarios para realizar el servicio. 


\section{RESULTADOS DEL ESTUDIO PARA EL SISTEMA DE RECOGIDA MEDIANTE CARGA LATERAL}

A continuación recogemos en la tabla 7.1 la información necesaria para deducir los recorridos a establecer para la recogida de materia orgánica y su transporte a la planta de compostaje.

TABLA 7.1

\begin{tabular}{|l|c|r|r|}
\hline & Kg. / día RSU & $\begin{array}{c}\text { No contenedores } \\
(2,4 \mathrm{~L} .)\end{array}$ & $\mathrm{m}^{3}$ caja compactadora \\
\hline BAEZA & 8051,3 & 31 & 10,4 \\
\hline BEGÍJAR & 1643,53 & 7 & 2,4 \\
\hline CANENA & 1111,4 & 5 & 1,6 \\
\hline IBROS & 1579,5 & 7 & 2,4 \\
\hline LUPIÓN & 526,7 & 2 & 0,8 \\
\hline RUS & 1984,31 & 8 & 2,8 \\
\hline SABIOTE & 2164,15 & 8 & 2,8 \\
\hline TORRE PEROGIL & 3616,32 & 14 & 4,8 \\
\hline ÚBEDA & 17950,95 & 71 & 23,6 \\
\hline TOTAL & 38610,61 & 153 & 51,6 \\
\hline
\end{tabular}

FUENTE: Elaboración propia, en base a encuestas realizadas

Como observamos en la tabla 7.1 el número de contenedores es mayor que las necesidades reales diarias ya que se ha tenido en cuenta que el servicio no se presta durante todos los días de la semana puesto que ello implicaría un incremento considerable de los costes. A continuación en las tablas 7.2 y 7.3 estableceremos los servicios de recogida: 
TABLA 7.2

\begin{tabular}{|l|c|c|c|}
\hline & Km. / viaje & no viajes & Total Km. semana \\
\hline BAEZA & 671,6 & 6 & 4029,6 \\
\hline BEGÍJAR & & & \\
\hline CANENA & & & \\
\hline IBROS & & & \\
\hline LUPIÓN & & & \\
\hline RUS & 1020.82 & 6 & 6124.92 \\
\hline SABIOTE & & & \\
\hline TORRE PEROGIL & & & \\
\hline ÚBEDA & & & \\
\hline
\end{tabular}

FUENTE: Elaboración propia

TABLA 7.3

\begin{tabular}{|l|r|r|r|r|r|r|}
\hline & $\begin{array}{r}\mathrm{m}^{3} \text { caja } \\
\text { compactadora }\end{array}$ & $\begin{array}{r}\mathrm{m}^{3} / \text { día } \\
\text { desplazamiento }\end{array}$ & $\begin{array}{r}\text { Horas } \\
\text { semana }\end{array}$ & Turnos & $\begin{array}{r}\text { Horas para } \\
\text { completar } \\
\text { servicio }\end{array}$ & $\begin{array}{r}\text { Horas para } \\
\text { completar } \\
\text { turno }\end{array}$ \\
\hline BAEZA & 10,24 & 15 & 90 & 2 & 20 & \\
\hline BEGIJAR & 2,09 & & & & & \\
\hline CANENA & 2 & & & & & \\
\hline IBROS & 0,67 & & & & & \\
\hline LUPIÓN & 1,41 & & & & & \\
\hline RUS & 2,52 & & & & & \\
\hline SABIOTE & 2,75 & 34,12 & 136 & 4 & & \\
\hline TORRE PEROGIL & 4,6 & & & & & \\
\hline ÚBEDA & 22,84 & & & & & \\
\hline
\end{tabular}

FUENTE: Elaboración propia

A la vista de los resultados expuestos en la tablas 7.2 y 7.3 podemos dividir el servicio en dos zonas una sombreada con verde que corresponderá a los 
municipios de Baeza, BEGÍJAR, Canena, Ibros, Lupión y Rus y la otra sombreada en naranja que agrupará a los municipios de Sabiote, Torre Perogil y Ú beda. El servicio se estructurará en 6 turnos y las 16 horas que restan deberán completarse con horas extras del personal o la contratación de un conductor a tiempo parcial. Así mismo, para la prestación del servicio se deberán adquirir 6 camiones que trabajarán en turno doble con el fin de minimizar los costes de inversión en vehículos.

\section{RESULTADOS DEL ESTUDIO PARA EL SISTEMA DE RECOGIDA ME- DIANTE CARGA TRASERA}

A continuación recogemos en la tabla 7.1 la información necesaria para deducir los recorridos a establecer para la recogida de materia orgánica y su transporte a la planta de compostaje.

TABLA 7.4

\begin{tabular}{|l|c|c|r|}
\hline & Kg. / día RSU & $\begin{array}{r}\text { No contenedores } \\
(2,4 \mathrm{~L} .)\end{array}$ & m³ caja compactadora \\
\hline BAEZA & 8051,3 & 67 & 10,4 \\
\hline BEGÍJAR & 1643,53 & 14 & 2,4 \\
\hline CANENA & 1111,4 & 9 & 1,6 \\
\hline IBROS & 1579,5 & 13 & 2,4 \\
\hline LUPIÓN & 526,7 & 4 & 0,8 \\
\hline RUS & 1984,31 & 16 & 2,8 \\
\hline SABIOTE & 2164,15 & 19 & 2,8 \\
\hline TORRE PEROGIL & 3616,32 & 30 & 4,8 \\
\hline ÚBEDA & 17950,95 & 150 & 23,6 \\
\hline TOTAL & 38610,61 & 322 & 51,6 \\
\hline
\end{tabular}

FUENTE: Elaboración propia, en base a encuestas realizadas

Como observamos en la tabla 7.4 el número de contenedores es mayor que las necesidades reales diarias ya que se ha tenido en cuenta que el servicio no se presta durante todos los días de la semana puesto que ello implica- 
ría un incremento considerable de los costes. A continuación en las tablas 7.5 y 7.6 estableceremos los servicios de recogida:

TABLA 7.5

\begin{tabular}{|l|r|r|r|r|}
\hline & $\begin{array}{r}\mathrm{m}^{3} \text { caja } \\
\text { compactadora }\end{array}$ & $\mathrm{Km} . /$ viaje & $\mathrm{n}$ - viajes & Total Km. semana \\
\hline BAEZA & 10,24 & 2491,87 & 6 & 14951,22 \\
\hline BEGÍJAR & 2,09 & & & \\
\hline IBROS & 2 & & & \\
\hline ÚBEDA & 22,84 & & & \\
\hline LUPIÓN & 0,67 & & & \\
\hline CANENA & 1,41 & 1465,33 & 6 & \\
\hline RUS & 2,52 & & & \\
\hline SABIOTE & 2,75 & & & \\
\hline TORRE PEROGIL & 4,6 & & & \\
\hline
\end{tabular}

FUENTE: Elaboración propia

TABLA 7.6

\begin{tabular}{|l|c|r|r|r|r|r|}
\hline & $\begin{array}{r}\mathrm{m}^{3} \text { caja } \\
\text { compactadora }\end{array}$ & $\begin{array}{r}\mathrm{m}^{3} / \text { día } \\
\text { desplazamiento }\end{array}$ & $\begin{array}{r}\text { Horas } \\
\text { semana }\end{array}$ & Turnos & $\begin{array}{r}\text { Horas para } \\
\text { completar } \\
\text { servicio }\end{array}$ & $\begin{array}{r}\text { Horas para } \\
\text { completar } \\
\text { turno }\end{array}$ \\
\hline BAEZA & 37,84 & 332,25 & 5 & 10 & 18 & \\
\hline BEGIJAR & & & & & & \\
\hline IBROS & & & & & & \\
\hline ÚBEDA & & & & & & \\
\hline LUPIÓN & & & & & & \\
\hline CANENA & 11,28 & 195 & 3 & 5 & & \\
\hline RUS & & & & & & \\
\hline SABIOTE & & & & & & \\
\hline TORRE PEROGIL & & & & & & \\
\hline
\end{tabular}

FUENTE: Elaboración propia 
A la vista de los resultados expuestos en la tablas 7.5 y 7.6 podemos dividir el servicio en dos zonas una sombreada con verde que corresponderá a los municipios de Sabiote, Torre Perogil, Canena y Rus y la otra sombreada en gris que agrupará a los municipios de Baeza, BEGÍJAR, Ibros, Lupión y Ú beda. El servicio se estructurará en 15 turnos y las 2 horas que restan deberán completarse con horas extras del personal. Así mismo, para la prestación del servicio se deberán adquirir 8 camiones que trabajarán en turno doble con el fin de minimizar los costes de inversión en vehículos.

\section{COSTES DE RECOGIDA Y TRANSPORTE}

A continuación comparamos mediante la tabla 8.1 los costes de los sistemas de recogida y transporte de residuos (materia orgánica) mediante carga lateral y trasera para la zona de estudio para su elaboración se ha tenido en cuenta que el salario de un conductor estaría en torno a $1450 €$ y el de un operario sobre los $1140 €^{7}$ y que el coste un contenedor de carga lateral de 2400 litros tiene es $917.85 €^{8}$ mientras que uno de carga trasera de 1100 litros cuesta $423.32 €^{9}$.

TABLA 8.1

Tabla comparativa de costes de los sistemas recogida de carga lateral y trasera en la comarca de estudio

\begin{tabular}{|l|l|l|}
\hline & CARGA LATERAL & CARGA TRASERA \\
\hline № contenedores & 153 & 322 \\
\hline COSTE DE LOS CONTENEDORES & $140390 €$ & $136310 €$ \\
\hline $\mathrm{N} N$ camiones & 6 & 8 \\
\hline COSTES EN VEHÍCULOS & $1080000 €$ & $960000 €$ \\
\hline № conductores & 6.5 & 15 \\
\hline № de operarios & 0 & 30 \\
\hline COSTES SALARIALES & $1319500 €$ & $7833000 €$ \\
\hline COSTES TOTALES & $2539890 €$ & $8929310 €$ \\
\hline
\end{tabular}

FUENTE: Elaboración propia, en base a encuestas realizadas

Según lo observado en la tabla 8.1 los costes globales del sistema de recogida de carga trasera serían 3.5 veces superior al de carga lateral debido 
principalmente a los costes salariales ya que la carga trasera requiere mayor número de trabajadores mientras que la inversión en vehículos y contenedores es inferior a la que tendríamos que realizar para el sistema de recogida mediante carga lateral.

En conclusión, para la zona de estudio sería más ventajoso desde el punto de vista económico el sistema de recogida mediante carga lateral.

\section{BIBLIOGRAFÍA}

(1). E. HONTORIA GARCÍA. Fundamentos de manejo de residuos urbanos, Madrid, Colegio de Ingenieros de Caminos, Canales y Puertos.. 2000, Página 266.

(2). M. GARCÍA RAMOS, "Gestión integral de los residuos de envases", Residuos no 32. 1996.

(3, 4 y 7). Datos obtenidos de consultas directas a la empresa CESPA-INGENIERÍA URBANA.

(5 y 6). Datos obtenidos de consultas directas a la empresa ROS-ROCA.

(8). Datos obtenidos de consultas directas a la empresa PLASTIC OMNIUM SISTEMAS URBANOS, S.A.

(9). Datos obtenidos de internet mediante la siguiente página web http:/ / es.logismarket.com/ naviagation/ product/ detail.jsp?id=3789369 $\underline{\alpha c t g}=1124850$ 